\title{
Psychiatry is Neurology: White matter pathology permeates psychiatric disorders
}

\section{Ask neurologists or psychiatrists} to name a white matter (WM) brain disease and they are very likely to say multiple sclerosis (MS), a demyelinating brain disorder caused by immune-mediated destruction of oligodendrocytes, the glial cells that manufacture myelin without which brain communications would come to a standstill.

MS is often associated with mood or psychotic disorders, yet it is regarded as a neurologic illness, not a psychiatric disorder.

Many neurologists and psychiatrists may not be aware that during the past few years, multiple diffusion tensor imaging (DTI) studies have revealed that many psychiatric disorders are associated with WM pathology. ${ }^{1}$

Most people think that the brain is composed mostly of neurons, but in fact the bulk of brain volume $(60 \%)$ is comprised of WM and only $40 \%$ is gray matter, which includes both neurons and glial cells (astroglia, microglia, and oligodendroglia). WM includes $>137,000$ $\mathrm{km}$ of myelinated fibers, an extensive network that connects all brain regions and integrates its complex, multifaceted functions, culminating in a unified sense of self and agency.

\section{The role of the corpus callosum}

Early in my research career, I became interested in the corpus callosum, the largest interhemispheric WM commissure connecting homologous areas across the 2 cerebral hemispheres. It is comprised of 200 million fibers of various diameters. Reasons for my fascination with the corpus callosum were:

The studies of Roger Sperry, the 1981 Nobel Laureate who led the team that was awarded the prize for split-brain research, which involved patients whose corpus callosum was cut to prevent the transfer of intractable epilepsy from 1 hemisphere to the other. Using a tachistoscope that he designed, Sperry discovered that the right and left hemispheres are 2 independent spheres of consciousness (ie, 2 individuals) with different skills. ${ }^{2}$ Cerebral dominance (laterality) fully integrates the 2 hemispheres via the corpus callosum, with a verbal hemisphere (the left, in $90 \%$ of people) dominating the other hemisphere and serving as the "spokesman self." Thus, we all have 2 persons in our brain completely integrated into 1 "self." 2 This led me to wonder about the effects of an impaired corpus callosum on the "unified self."

Postmortem and MRI studies conducted by our research group showed a significant difference in the thickness of the corpus callosum in a group of patients

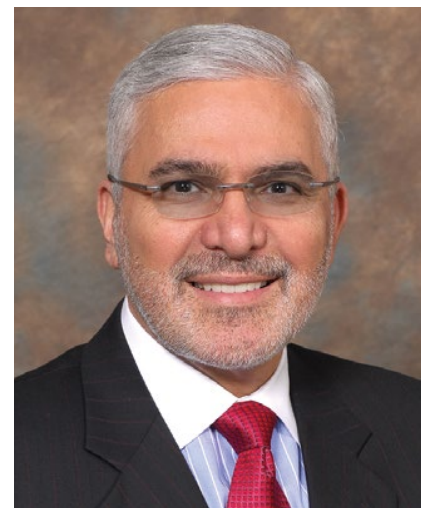

Henry A. Nasrallah, MD Editor-in-Chief doi: 10.12788/cp.0138

\section{Multiple diffusion} tensor imaging studies have revealed that many psychiatric disorders are associated with white matter pathology
To comment on this editorial or other topics of interest: henry.nasrallah @currentpsychiatry.com 
Editorial Staff

EDITOR Jeff Bauer

SENIOR EDITOR Sathya Achia Abraham

ASSISTANT EDITOR Matthew Wyles

WEB EDITOR Kathryn Wighton

Art \& Production Staff

CREATIVE DIRECTOR Louise Koenig

ART DIRECTOR Pat Fopma

DIRECTOR, JOURNAL MANUFACTURING

Michael Wendt

PRODUCTION MANAGER Donna Pituras

Publishing Staff

PUBLISHER Sharon Finch

DIRECTOR EBUSINESS DEVELOPMENT

Alison Paton

SENIOR DIRECTOR OF SALES

Tim LaPella

Editor-in-Chief Emeritus

James Randolph Hillard, MD

Frontline Medical Communications

VP, SALES Mike Guire

VP, DIGITAL CONTENT \& STRATEGY

Amy Pfeiffer

PRESIDENT, CUSTOM SOLUTIONS

JoAnn Wahl

CIRCULATION DIRECTOR Jared Sonners

In affiliation with Global Academy for Medical Education, LLC

PRESIDENT David J. Small, MBA

\section{FRONTLNNE MLedge}

7 Century Drive, Suite 302

Parsippany, NJ 07054

Tel: (973) 206-3434

Fax: (973) 206-9378

www.frontlinemedcom.com

Subscription Inquiries:

subscriptions@mdedge.com

\section{Table 1}

\section{High-yield diffusion tensor imaging terms}

\begin{tabular}{l|l} 
Measurement & Definition \\
\hline Fractional anisotropy (FA) & $\begin{array}{l}\text { Values range between } 0 \text { (water motion random) } \\
\text { and 1 (directional selectivity of water movement) }\end{array}$ \\
\hline Mean diffusivity (MD) & Average molecule motion, regardless of direction \\
\hline Axial diffusivity (AD) & Molecule movement parallel to axons \\
\hline Radial diffusivity (RD) & Molecule movement perpendicular to axons \\
\hline
\end{tabular}

\section{Table 2}

\section{Psychiatric disorders and symptoms reported to have white matter pathology}

\begin{tabular}{|c|}
\hline Schizophrenia $^{7}$ \\
\hline Bipolar disorder ${ }^{8}$ \\
\hline Major depressive disorder ${ }^{9}$ \\
\hline Obsessive-compulsive disorder ${ }^{10}$ \\
\hline Anxiety disorders ${ }^{11}$ \\
\hline Autism spectrum disorder ${ }^{12}$ \\
\hline Alcohol use disorders ${ }^{13}$ \\
\hline Substance use disorders ${ }^{14}$ \\
\hline Borderline personality disorder ${ }^{15}$ \\
\hline Antisocial personality ${ }^{16}$ \\
\hline Psychopathy ${ }^{17}$ \\
\hline Conduct disorder ${ }^{18}$ \\
\hline Oppositional defiant disorder ${ }^{19}$ \\
\hline
\end{tabular}

with schizophrenia vs healthy controls, which implied abnormal connectivity across the left and right hemispheres. ${ }^{3}$

I then conducted a clinical study examining patients with tumors impinging on the corpus callosum, which revealed that they developed psychotic symptoms (delusions and hallucinations). ${ }^{4}$ This study suggested that disrupting the integrity of the callosal inter-hemispheric fibers can trigger fixed false beliefs and perceptual anomalies. ${ }^{4}$

\section{A 'dysconnection' between hemispheres}

I translated those observations about the corpus callosum into a published hypothesis ${ }^{5}$ in which I proposed that
Schneider's First-Rank Symptoms of schizophrenia of thought insertion, thought withdrawal, and thought broadcasting-as well as delusional experiences of "external control"-may be due to a neurobiologic abnormality in the corpus callosum that disrupts the flow of ongoing bits of information transmitted from the left to the right hemisphere, and vice versa. I proposed in my model that this disruption leads to the verbal left hemisphere of a psychotic patient to describe having thoughts inserted into it from an alien source, failing to recognize that the thoughts it is receiving are being transmitted from the disconnected right hemisphere, which is no longer part of the "self." Similarly, impulses from the right hemispheric consciousness are now perceived by the patient's verbal left hemisphere (which talks to the examining physician) as "external control." Thus, I postulated that an abnormal corpus callosum structure would lead to a "dysconnection" (not "disconnection") between the 2 hemispheres, and that anomalous dysconnectivity may generate both delusions and hallucinations. ${ }^{6}$

Two decades later, my assumptions were vindicated when DTI was invented, enabling the measurement of WM integrity, including the corpus callosum, the largest body of WM in the brain. Table 1 defines the main parameters of WM integrity, anisotropy and diffusivity, which measure water flow inside WM fibers. 


\section{Neuroprotective and promyelination effects of prolactin}

\begin{tabular}{l}
\hline Enhances and sustains neurogenesis \\
\hline Protects from damage caused by seizures \\
\hline Protects against glutamate neurotoxicity \\
\hline Protects the retina from degeneration \\
\hline Stimulates immune response \\
\hline Protects against apoptosis
\end{tabular}

Promyelination effects: Repairs white matter damage by stimulating the proliferation of oligodendrocytes, the main source of myelin in the brain (and the number of which declines in schizophrenia and related psychoses)

If women who have multiple sclerosis (MS) become pregnant, their MS may go into remission as their prolactin rises dramatically. The remission will continue during lactation, and relapse occurs when they wean their babies and prolactin drops precipitously

Source: References 22,23

During the past 15 years, many studies have confirmed the presence of significant abnormalities in the myelinated fibers of the corpus callosum in schizophrenia, which can be considered a validation of my hypothesis that the corpus callosum becomes a dysfunctional channel of communications between the right and left hemisphere. Subsequently, DTI studies have reported a spectrum of WM pathologies in various other cerebral bundles and not only in schizophrenia, but also in other major psychiatric disorders (Table 2,-19 page 8 ).

The pathophysiology of WM pathology in many psychiatric disorders may include neurodevelopmental aberrations (genetic, environmental, or both, which may alter WM structure and/or myelination), neuroinflammation, or oxidative stress (free radicals), which can cause disintegration of the vital myelin sheaths, leading to disruption of brain connectivity, ${ }^{6,7}$ Researchers now consider the brain's WM network dysconnectivity as generating a variety of psychiatric symptoms, including psychosis, depression, mania, anxiety, autism, aggression, impulsivity, psychopathy, and cognitive impairments.
It is not surprising that $\mathrm{WM}$ repair has become a therapeutic target in psychiatry and neurology. Among the strategies being investigated are inhibiting the Nogo-A signaling pathways ${ }^{20}$ or modulating the Lingo-1 signaling. ${ }^{21}$ However, the most well-established myelin repair pathway is prolactin, a neuroprotective hormone with several beneficial effects on the brain (Table $3^{22,23}$ ), including the proliferation of oligodendroglia, the main source of myelin (and the number of which declines in schizophrenia). Antipsychotics that increase prolactin have been shown to increase WM volume..$^{24,25}$ It has even been proposed that a decline in oligodendrocytes and low myelin synthesis may be one of the neurobiologic pathologies in schizophrenia. ${ }^{26}$ One of the 24 neuroprotective properties of the second-generation antipsychotics (SGAs) is the restoration of WM integrity. ${ }^{27}$ It's worth noting that WM pathology has been found to be present at the onset of schizophrenia before treatment, and that SGAs have been reported to correct it. ${ }^{28}$

In conclusion, psychiatric disorders, usually referred to as "mental illnesses," are unquestionably neurologic disorders. Similarly, all neurologic
The pathophysiology of WM pathology in many psychiatric disorders may include neurodevelopmental aberrations, neuroinflammation, or oxidative stress 
I strongly advocate that psychiatry and neurology reunite into a single medical specialty disorders are associated with psychiatric manifestations. WM pathology is only 1 of numerous structural brain abnormalities that have been documented across psychiatric disorders, which proves that psychiatry is a clinical neuroscience, just like neurology. I strongly advocate that psychiatry and neurology reunite into a single medical specialty. Both focus on disorders of brain structure and/or function, and these disorders also share much more than WM pathology. ${ }^{29}$

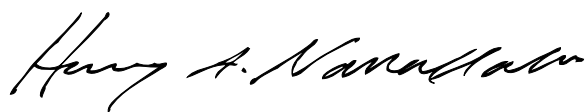

\section{Henry A. Nasrallah, MD}

Editor-in-Chief

\section{References}

1. Sagarwala $\mathrm{R}$ and Nasrallah HA. White matter pathology is shared across multiple psychiatric brain disorders: Is abnormal diffusivity a transdiagnostic biomarker for psychopathology? Biomarkers in Neuropsychiatry. 2020;2:00010. https://doi.org/ 10.1016/j.bionps.2019.100010

2. Pearce JMS; FRCP. The "split brain" and Roger Wolcott Sperry (1913-1994). Rev Neurol (Paris). 2019;175(4):217-220

3. Nasrallah HA, Andreasen NC, Coffman JA, et al. A controlled magnetic resonance imaging study of corpus callosum thickness in schizophrenia. Biol Psychiatry. 1986;21(3):274-282.

4. Nasrallah HA, McChesney CM. Psychopathology of corpus callosum tumors. Biol Psychiatry. 1981; 16(7):663-669.

5. Nasrallah HA. The unintegrated right cerebral hemispheric consciousness as alien intruder: a possible mechanism for Schneiderian delusions in schizophrenia. Compr Psychiatry. 1985;26(3):273-282.

6. Friston $\mathrm{K}$, Brown HR, Siemerkus J, et al. The dysconnection hypothesis (2016). Schizophr Res. 2016;176(2-3):83-94.

7. Najjar S, Pearlman DM. Neuroinflammation and white matter pathology in schizophrenia: systematic review. Schizophr Res. 2015;161(1):102-112.

8. Benedetti F, Bollettini I. Recent findings on the role of white matter pathology in bipolar disorder. Harv Rev Psychiatry. 2014;22(6):338-341.

9. Zheng H, Bergamino M, Ford BN, et al; Tulsa 1000 Investigators. Replicable association between human cytomegalovirus infection and reduced white matter fractional anisotropy in major depressive disorder. Neuropsychopharmacology. 2021;46(5):928-938.

10. Sagarwala R, Nasrallah HA. A systematic review of diffusion tensor imaging studies in drug-naïve OCD patients before and after pharmacotherapy. Ann Clin Psychiatry. 2020;32(1):42-47.

11. Lee KS, Lee SH. White matter-based structural brain network of anxiety. Adv Exp Med Biol. 2020;1191: 61-70.

12. Swanson MR, Hazlett HC. White matter as monitoring biomarker for neurodevelopmental
disorderinterventionstudies.JNeurodev Disord.2019; 11(1):33

13. Hampton WH, Hanik IM, Olson IR. Substance abuse and white matter: findings, limitations, and future of diffusion tensor imaging research. Drug Alcohol Depend. 2019;197:288-298.

14. Waller R, Dotterer HL, Murray L, et al. Whitematter tract abnormalities and antisocial behavior: systematic review of diffusion tensor imaging studies across development. Neuroimage Clin. 2017;14: 201-215.

15. Wolf RC, Pujara MS, Motzkin JC, et al. Interpersonal traits of psychopathy linked to reduced integrity of the uncinate fasciculus. Hum Brain Mapp. 2015; 36(10):4202-4209.

16. Puzzo I, Seunarine K, Sully K, et al. Altered white-matter microstructure in conduct disorder is specifically associated with elevated callousunemotional traits. J Abnorm Child Psychol. 2018, 46(7):1451-1466.

17. Finger EC, Marsh A, Blair KS, et al. Impaired functional but preserved structural connectivity in limbic white matter tracts in youth with conduct disorder or oppositional defiant disorder plus psychopathic traits. Psychiatry Res. 2012;202(3):239-244.

18. Li C, Dong M, Womer FY, et al. Transdiagnostic timevarying dysconnectivity across major psychiatric disorders. Hum Brain Mapp. 2021;42(4):1182-1196.

19. Khanbabaei M, Hughes E, Ellegood J, et al. Precocious myelination in a mouse model of autism. Transl Psychiatry. 2019;9(1):251.

20. Petratos S, Theotokis P, Kim MI, et al. That's a wrap Molecular drivers governing neuronal nogo receptordependent myelin plasticity and integrity. Front Cell Neurosci. 2020;14:227

21. Fernandez-Enright F, Andrews JL, Newell KA, et al. Novel implications of Lingo-1 and its signaling partners in schizophrenia. Transl Psychiatry. 2014; 4(1):e348. doi: 10.1038/tp.2013.121

22. Bartzokis G, Lu PH, Stewart SB, et al. In vivo evidence of differential impact of typical and atypical antipsychotics on intracortical myelin in adults with schizophrenia. Schizophr Res. 2009;113(2-3):322-331.

23. Bartzokis G, Lu PH, Amar CP, et al. Long acting injection versus oral risperidone in first-episode schizophrenia: differential impact on white matter myelination trajectory. Schizophr Res. 2011 Oct;132(1):35-41

24. Tishler TA, Bartzokis G, Lu PH, et al. Abnormal trajectory of intracortical myelination in schizophrenia implicates white matter in disease pathophysiology and the therapeutic mechanism of action of antipsychotics. Biol Psychiatry Cogn Neurosci Neuroimaging. 2018;3(5):454-462

25. Ren $\mathrm{Y}$, Wang $\mathrm{H}$, Xiao L. Improving myelin/ oligodendrocyte-related dysfunction: a new mechanism of antipsychotics in the treatment of schizophrenia? Int J Neuropsychopharmacol. 2013, 16(3):691-700.

26. Dietz AG, Goldman SA, Nedergaard M. Glial cells in schizophrenia: a unified hypothesis. Lancet Psychiatry. 2020;7(3):272-281.

27. Chen AT, Nasrallah HA. Neuroprotective effects of the second generation antipsychotics. Schizophr Res. 2019;208:1-7

28. Sagarwala R, Nasrallah HA. (In press.) The effect of antipsychotic medications on white matter integrity in first-episode drug naïve patients with psychosis. Asian Journal of Psychiatry.

29. Nasrallah HA. Let's tear down the silos and reunify psychiatry and neurology. Current Psychiatry. 2013 12(8):9-10. 